J. Korean Math. Soc. 52 (2015), No. 2, pp. 417-429

http://dx.doi.org/10.4134/JKMS.2015.52.2.417

\title{
THE ANNIHILATOR IDEAL GRAPH OF A COMMUTATIVE RING
}

\author{
Abolfazl Alibemani, Moharram Bakhtyiari, Reza Nikandish, \\ and Mohammad Javad Nikmehr
}

\begin{abstract}
Let $R$ be a commutative ring with unity. The annihilator ideal graph of $R$, denoted by $\Gamma_{\mathrm{Ann}}(R)$, is a graph whose vertices are all non-trivial ideals of $R$ and two distinct vertices $I$ and $J$ are adjacent if and only if $I \cap \operatorname{Ann}(J) \neq\{0\}$ or $J \cap \operatorname{Ann}(I) \neq\{0\}$. In this paper, we study some connections between the graph-theoretic properties of this graph and some algebraic properties of rings. We characterize all rings whose annihilator ideal graphs are totally disconnected. Also, we study diameter, girth, clique number and chromatic number of this graph. Moreover, we study some relations between annihilator ideal graph and zero-divisor graph associated with $R$. Among other results, it is proved that for a Noetherian ring $R$ if $\Gamma_{\mathrm{Ann}}(R)$ is triangle free, then $R$ is Gorenstein.
\end{abstract}

\section{Introduction}

Recently, using graph theoretical tools in the investigation of rings attracted many researchers. There are many papers on assigning a graph to rings, see for example [1], [2], [6], [7] and [10]. When one assigns a graph to an algebraic structure numerous interesting algebraic problems arise from the translation of some graph-theoretic parameters such as clique number, chromatic number, independence number and so on. The main purpose of this paper is to introduce and study a new graph-annihilator ideal graph-associated with a ring.

Throughout this paper $R$ is a commutative ring with unity. The sets of all zero-divisors, nilpotent elements, non-trivial ideals, minimal prime ideals, maximal ideals, jacobson radical and the set of prime ideals of $R$ are denoted by $Z(R), \operatorname{Nil}(R), \mathbb{I}(R), \operatorname{Min}(R), \operatorname{Max}(R), \mathrm{J}(R)$ and $\operatorname{Spec}(R)$, respectively. Also, we denote by $\mathbb{Z}_{n}$ and $Z(M)$ the integers modulo $n$ and the set of all zerodivisors of an $R$-module $M$. A non-zero ideal $I$ of $R$ is called essential, denoted by $I \leq_{e} R$, if $I$ has a non-zero intersection with any non-zero ideal of $R$. The ring $R$ is said to be reduced if it has no non-zero nilpotent element. The socle of an $R$-module $M$, denoted by $\operatorname{soc}(M)$, is the sum of all simple submodules

Received June 24, 2014; Revised September 24, 2014.

2010 Mathematics Subject Classification. 13A99, 05C75, 05C69.

Key words and phrases. annihilator ideal graph, diameter, Clique number. 
of $M$. If there are no simple submodules, this sum is defined to be zero. It is well known $\operatorname{soc}(M)$ is the intersection of all essential submodules (see [13, 21.1]). By $\operatorname{dim}(R)$ and $\operatorname{depth}(R)$, we mean the dimension and depth of $R$, see [11]. We write $\operatorname{depth}(R)=0$ if and only if every non-unit element of a ring $R$ is zero-divisor. We say $x$ is a regular element of $R$ if $x$ is non-unit and non zero-divisor.

Let $G=(V, E)$ be a graph, where $V=V(G)$ is the set of vertices and $E=$ $E(G)$ is the set of edges. By $\bar{G}, \operatorname{diam}(G)$ and $\operatorname{gr}(G)$, we mean the complement, the diameter and the girth of $G$, respectively. Also, for a vertex $v \in V$, the degree of $v$ is denoted by $\operatorname{deg}(v)$ and the maximum degree in a graph $G$ denoted by $\Delta(G)$. The graph $H=\left(V_{0}, E_{0}\right)$ is a subgraph of $G$ if $V_{0} \subseteq V$ and $E_{0} \subseteq E$. Moreover, $H$ is called an induced subgraph by $V_{0}$, if $V_{0} \subseteq V$ and $E_{0}=\{\{u, v\} \in$ $\left.E \mid u, v \in V_{0}\right\}$. For two vertices $u$ and $v$ in $G$, the notation $u-v$ means that $u$ and $v$ are adjacent. A graph $G$ is said to be totally disconnected if it has no edge. The chromatic number of $G$, denoted by $\chi(G)$, is the minimal number of colors which can be assigned to the vertices of $G$ in such a way that every two adjacent vertices have different colors. A complete bipartite graph with part sizes $m$ and $n$ is denoted by $K_{m, n}$. If the size of one of the parts is 1 , then the graph is said to be a star graph. A clique of $G$ is a complete subgraph of $G$ and the number of vertices in a largest clique of $G$, denoted by $\omega(G)$, is called the clique number of $G$. In a graph $G$, a set $S \subseteq V(G)$ is an independent set if the subgraph induced by $S$ is totally disconnected. The independence number $\alpha(G)$ is the maximum size of an independent set in $G$. A forest is a graph with no cycle. Let $G_{1}$ and $G_{2}$ be two disjoint graphs. The join of $G_{1}$ and $G_{2}$, denoted by $G_{1} \vee G_{2}$, is a graph with the vertex set $V\left(G_{1} \vee G_{2}\right)=V\left(G_{1}\right) \cup V\left(G_{2}\right)$ and edge set $E\left(G_{1} \vee G_{2}\right)=E\left(G_{1}\right) \cup E\left(G_{2}\right) \cup\left\{u v \mid u \in V\left(G_{1}\right), v \in V\left(G_{2}\right)\right\}$.

Let $R$ be a commutative ring with $1 \neq 0$. Authors in [2], introduced the zero-divisor graph of $R$, denoted by $\Gamma(R)$, as the graph with the vertex set $Z^{*}(R)=Z(R) \backslash\{0\}$, and two distinct vertices $x$ and $y$ are adjacent if and only if $x y=0$. The annihilator ideal graph of $R$, denoted by $\Gamma_{\text {Ann }}(R)$, is a graph whose vertices are all non-trivial ideals of $R$ and two distinct vertices $I$ and $J$ are adjacent if and only if $I \cap \operatorname{Ann}(J) \neq\{0\}$ or $J \cap \operatorname{Ann}(I) \neq\{0\}$. Since the most properties of a ring are closely tied to the behavior of its ideals, one may expect that the annihilator ideal graph of a ring reflects many properties of a ring.

\section{The diameter and girth of $\Gamma_{\text {Ann }}(R)$}

In this section, we study the diameter and the girth of the annihilator ideal graph of a ring. It is proved that if $\Gamma_{\text {Ann }}(R)$ is connected, then $\operatorname{diam}\left(\Gamma_{\text {Ann }}(R)\right)$ $\leq 2$. Also if $\Gamma_{\text {Ann }}(R)$ contains a cycle, then $\operatorname{gr}\left(\Gamma_{\text {Ann }}(R)\right) \leq 3$. Finally, we investigate some relations between the diameters of $\Gamma_{\mathrm{Ann}}(R)$ and $\Gamma(R)$.

Lemma 1. Let $R$ be a ring. Then $\Gamma_{\mathrm{Ann}}(R)$ is totally disconnected if and only if either $R$ is an integral domain or $R$ has only one non-zero proper ideal. 
Proof. One side is clear. To prove the other side, suppose that $\Gamma_{\text {Ann }}(R)$ is totally disconnected with at least two vertices. We show that $R$ is an integral domain. Assume to the contrary, $x$ is a non-zero zero-divisor in $R$. Clearly $\operatorname{Ann}(x) \neq\{0\}$. Let $I \in \mathbb{I}(R) \backslash\{\operatorname{Ann}(x)\}$. If $I \cap \operatorname{Ann}(x)=\{0\}$, then $I$ is adjacent to $\operatorname{Ann}(x)$, which is impossible. Thus suppose that there exists a nonzero element in $I \cap \operatorname{Ann}(x)$, say $y$. If $R x=R y$, then $I$ is adjacent to $\operatorname{Ann}(x)$ and if $R x \neq R y$, then $R x$ is adjacent to $R y$. So, $\Gamma_{\text {Ann }}(R)$ contains an edge, a contradiction. Therefore, $R$ is an integral domain.

In the next theorem, it is proved that if $R$ is not an integral domain, then $\Gamma_{\text {Ann }}(R)$ is a connected graph of diameter at most 2 .

Theorem 2. Let $R$ be a ring. Then $\operatorname{diam}\left(\Gamma_{\text {Ann }}(R)\right) \in\{0,1,2, \infty\}$. In particular, if $R$ is not an integral domain, then $\Gamma_{\mathrm{Ann}}(R)$ is connected.

Proof. Suppose that $R$ is not an integral domain and $I, J$ are two non-adjacent vertices of $\Gamma_{\text {Ann }}(R)$. Since $I$ and $J$ are not adjacent, we deduce that $I J \neq\{0\}$. Let $x$ be a non-zero zero-divisor element in $I \cap J$ and $y \in \operatorname{Ann}(x)$. Now, if $R y, I$ and $J$ are distinct, then we find the path $I-R y-J$. Not that if $R y$ is equal to one of the ideals $I$ or $J$, then $I$ would be adjacent to $J$, which is impossible. Therefore, $\operatorname{diam}\left(\Gamma_{\text {Ann }}(R)\right) \in\{1,2\}$. Finally, if either $R$ is an integral domain or $R$ has only one non-zero proper ideal, then by Lemma 1, we have $\operatorname{diam}\left(\Gamma_{\text {Ann }}(R)\right) \in\{0, \infty\}$. Therefore, $\operatorname{diam}\left(\Gamma_{\text {Ann }}(R)\right) \in\{0,1,2, \infty\}$.

The next corollary is an immediate consequence of Theorem 2 .

Corollary 3. Suppose that $R$ is not an integral domain. Then $\Gamma_{\text {Ann }}(R)$ is a bipartite graph if and only if it is a complete bipartite graph.

To determine the girth of $\Gamma_{\mathrm{Ann}}(R)$, the following lemma is needed.

Lemma 4. Let $R$ be a non-reduced ring. Then there exists a vertex of $\Gamma_{\mathrm{Ann}}(R)$ which is adjacent to every other vertex.

Proof. Since $R$ is a non-reduced ring, we may assume that there exists a nontrivial ideal $I$ in $R$ such that $I^{2}=\{0\}$. We show that $I$ is adjacent to every other vertex. Let $J$ be a non-trivial ideal in $R$. If $I \cap J=\{0\}$, then clearly $I$ is adjacent to $J$. Otherwise, if $I \cap J \neq\{0\}$, then $I^{2}=\{0\}$ implies that $I$ is adjacent to $J$. This completes the proof.

Theorem 5. Let $R$ be a ring. Then $\operatorname{gr}\left(\Gamma_{\mathrm{Ann}}(R)\right) \in\{3, \infty\}$.

Proof. Suppose that $R$ is a non-reduced ring. Then by Lemma $4, \operatorname{gr}\left(\Gamma_{\mathrm{Ann}}(R)\right)$ $\in\{3, \infty\}$. Now assume that $R$ is a reduced ring. If $R$ is decomposable, then it is not hard to see that $\operatorname{gr}\left(\Gamma_{\text {Ann }}(R)\right) \in\{3, \infty\}$. Hence, we may assume that $R$ is indecomposable. Now, if $R$ is an integral domain, then by Lemma 1 , $\operatorname{gr}\left(\Gamma_{\text {Ann }}(R)\right)=\infty$. Otherwise, let $I \in \mathbb{I}(R)$ be such that $\operatorname{Ann}(I) \neq\{0\}$. Since $R$ is a reduced indecomposable ring, $\mathfrak{m}$ is essential and $\operatorname{Ann}(\mathfrak{m})=\{0\}$, for every $\mathfrak{m} \in \operatorname{Max}(R)$. Then $I-\operatorname{Ann}(I)-\mathfrak{m}-I$ is a triangle in $\Gamma_{\text {Ann }}(R)$, for some $\mathfrak{m} \in \operatorname{Max}(R)$. Therefore, the proof is complete. 
By [2, Theorem 2.3], $\Gamma(R)$ is connected and diam $(\Gamma(R)) \leq 3$, for every ring $R$. In the next theorem, we study some relations between the diameters of $\Gamma_{\text {Ann }}(R)$ and $\Gamma(R)$.

Theorem 6. Let $R$ be a ring. Then the following statements hold:

(i) If $\operatorname{diam}(\Gamma(R))=0$, then $\operatorname{diam}\left(\Gamma_{\mathrm{Ann}}(R)\right)=0$.

(ii) If $\operatorname{diam}(\Gamma(R))=1$, then $\operatorname{diam}\left(\Gamma_{\mathrm{Ann}}(R)\right)=0$ or 1 .

(iii) If $\operatorname{diam}(\Gamma(R))=2$, then $\operatorname{diam}\left(\Gamma_{\mathrm{Ann}}(R)\right)=1$ or 2 .

(iv) If $\operatorname{diam}(\Gamma(R))=3$, then $\operatorname{diam}\left(\Gamma_{\text {Ann }}(R)\right)=1$ or 2 .

(v) If $\operatorname{diam}\left(\Gamma_{\mathrm{Ann}}(R)\right)=0$ and $R$ is not an integral domain, then $\operatorname{diam}(\Gamma(R))=0$ or 1 .

(vi) If $\operatorname{diam}\left(\Gamma_{\mathrm{Ann}}(R)\right)=1$, then $\operatorname{diam}(\Gamma(R))=1$ or 2 or 3 .

(vii) If $\operatorname{diam}\left(\Gamma_{\mathrm{Ann}}(R)\right)=2$, then $\operatorname{diam}(\Gamma(R))=2$ or 3 .

Proof. (i) Suppose that $\operatorname{diam}(\Gamma(R))=0$. Then $|Z(R)|=2$ and so it is not hard to see that $R$ is isomorphic to one of the rings $\mathbb{Z}_{4}$ or $\frac{\mathbb{Z}_{2}[x]}{\left(x^{2}\right)}$. By Lemma 1 , $\operatorname{diam}\left(\Gamma_{\text {Ann }}(R)\right)=0$.

(ii) Suppose that $\operatorname{diam}(\Gamma(R))=1$. Then by [2, Theorem 2.8], $R \cong \mathbb{Z}_{2} \times \mathbb{Z}_{2}$ or $x y=0$ for all $x, y \in Z(R)$, and $R$ is not isomorphic to either $\mathbb{Z}_{4}$ or $\frac{\mathbb{Z}_{2}[x]}{\left(x^{2}\right)}$. If $R \cong \mathbb{Z}_{2} \times \mathbb{Z}_{2}$, then obviously $\operatorname{diam}\left(\Gamma_{\text {Ann }}(R)\right)=1$. Also, if $x y=0$ for all $x, y \in Z(R)$, and $R$ is not isomorphic to either $\mathbb{Z}_{4}$ or $\frac{\mathbb{Z}_{2}[x]}{\left(x^{2}\right)}$, then $\Gamma_{\text {Ann }}(R)$ is a complete graph and hence $\operatorname{diam}\left(\Gamma_{\text {Ann }}(R)\right) \leq 1$. Note that if $|\mathbb{I}(R)|=1$ (say $\mathbb{Z}_{p^{2}}$, where $p$ is a prime number), then $\operatorname{diam}\left(\Gamma_{\text {Ann }}(R)\right)=0$. Therefore, $\operatorname{diam}\left(\Gamma_{\text {Ann }}(R)\right)=0$ or 1 .

(iii) Suppose that $\operatorname{diam}(\Gamma(R))=2$. If $\operatorname{diam}\left(\Gamma_{\text {Ann }}(R)\right)=0$, then $R$ has only one non-trivial ideal $I$ such that $I^{2}=\{0\}$ (i.e., $Z(R)=I$ ). Thus $\Gamma(R)$ is a complete graph and hence $\operatorname{diam}(\Gamma(R))=0$ or 1 . Therefore, $\operatorname{diam}\left(\Gamma_{\text {Ann }}(R)\right)=$ 1 or 2 . Now we show that these two cases may occur. To see this, let $R_{1} \cong$ $\mathbb{Z}_{2} \times \mathbb{Z}$ and $R_{2} \cong \mathbb{Z}_{3} \times \mathbb{Z}_{3}$. Then it is not hard to see that $\operatorname{diam}\left(\Gamma\left(R_{1}\right)\right)=$ $\operatorname{diam}\left(\Gamma\left(R_{2}\right)\right)=2$, but $\operatorname{diam}\left(\Gamma_{\text {Ann }}\left(R_{1}\right)\right)=2$ and $\operatorname{diam}\left(\Gamma_{\text {Ann }}\left(R_{2}\right)\right)=1$.

(iv) Suppose that $\operatorname{diam}(\Gamma(R))=3$. Then there exist $x, y \in Z^{*}(R)$ such that $d(x, y)=3$. Let $x-a-b-y$ be the shortest path between $x, y$ in $\Gamma(R)$, where $a, b \in Z^{*}(R)$. If $R a=R b$, then $\operatorname{Ann}(R a)=\operatorname{Ann}(R b)$, which is a contradiction. Thus $R a \neq R b$ and so $\operatorname{diam}\left(\Gamma_{\text {Ann }}(R)\right)=1$ or 2 . Now we show that these two cases may happen. To see this, let $R_{1} \cong \mathbb{Z}_{2} \times \mathbb{Z}_{4}, R_{2} \cong \mathbb{Z}_{2} \times \frac{\mathbb{Z}_{2}\left[X_{1}, \ldots, X_{i}, \ldots\right]}{\left(X_{1}^{2}, \ldots, X_{i}^{2}, \ldots\right)}$. Then it is easy to see that $\operatorname{diam}\left(\Gamma\left(R_{1}\right)\right)=\operatorname{diam}\left(\Gamma\left(R_{2}\right)\right)=3$, but $\operatorname{diam}\left(\Gamma_{\text {Ann }}\left(R_{1}\right)\right)=1$ and $\operatorname{diam}\left(\Gamma_{\text {Ann }}\left(R_{2}\right)\right)=2$.

(v) Suppose that $\operatorname{diam}\left(\Gamma_{\text {Ann }}(R)\right)=0$ and $R$ is not an integral domain. Then $R$ has only one non-trivial ideal $I$ such that $I^{2}=\{0\}$ (i.e., $Z(R)=I$ ). Thus clearly $\Gamma(R)$ is a complete graph and hence $\operatorname{diam}(\Gamma(R))=0$ or 1 . These two cases may happen. Let $R_{1} \cong \mathbb{Z}_{4}$ and $R_{2} \cong \mathbb{Z}_{9}$. Then $\operatorname{diam}\left(\Gamma_{\mathrm{Ann}}\left(R_{1}\right)\right)=$ $\operatorname{diam}\left(\Gamma_{\text {Ann }}\left(R_{2}\right)\right)=0$, but $\operatorname{diam}\left(\Gamma\left(R_{1}\right)\right)=0$ and $\operatorname{diam}\left(\Gamma\left(R_{1}\right)\right)=1$. 
(vi) Suppose that $\operatorname{diam}\left(\Gamma_{\mathrm{Ann}}(R)\right)=1$. If $\operatorname{diam}(\Gamma(R))=0$, then $R$ is isomorphic to one of the rings $\mathbb{Z}_{4}$ or $\frac{\mathbb{Z}_{2}[x]}{\left(x^{2}\right)}$. Thus $\operatorname{diam}\left(\Gamma_{\text {Ann }}(R)\right)=0$, which is a contradiction. Hence $\operatorname{diam}(\Gamma(R))=1$ or 2 or 3 . These three cases may happen. If $R_{1} \cong \mathbb{Z}_{2} \times \mathbb{Z}_{2}, R_{2} \cong \mathbb{Z}_{6}$ and $R_{3} \cong \mathbb{Z}_{2} \times \mathbb{Z}_{4}$, then $\operatorname{diam}\left(\Gamma_{\text {Ann }}\left(R_{1}\right)\right)=$ $\operatorname{diam}\left(\Gamma_{\text {Ann }}\left(R_{2}\right)\right)=\operatorname{diam}\left(\Gamma_{\text {Ann }}\left(R_{3}\right)\right)=1, \operatorname{but} \operatorname{diam}\left(\Gamma\left(R_{1}\right)\right)=1, \operatorname{diam}\left(\Gamma\left(R_{2}\right)\right)=$ 2 and $\operatorname{diam}\left(\Gamma\left(R_{3}\right)\right)=3$.

(vii) Suppose that $\operatorname{diam}\left(\Gamma_{\mathrm{Ann}}(R)\right)=2$. Then by statements (a) and (b), $\operatorname{diam}(\Gamma(R))=2$ or 3 . Now we show that these two cases may occur. To see this, let $R_{1} \cong \mathbb{Z}_{2} \times \mathbb{Z}$ and $R_{2} \cong \mathbb{Z}_{2} \times \frac{\mathbb{Z}_{2}\left[X_{1}, \ldots, X_{i}, \ldots\right]}{\left(X_{1}^{2}, \ldots, X_{i}^{2}, \ldots\right)}$. Then $\operatorname{diam}\left(\Gamma_{\mathrm{Ann}}\left(R_{1}\right)\right)=$ $\operatorname{diam}\left(\Gamma_{\text {Ann }}\left(R_{2}\right)\right)=2$ and it easily seen that $\operatorname{diam}\left(\Gamma\left(R_{1}\right)\right)=2$ and $\operatorname{diam}\left(\Gamma\left(R_{2}\right)\right)$ $=3$.

\section{Rings whose annihilator ideal graphs are complete}

In this section, we study rings whose annihilator ideal graphs are complete. It is proved that if $R$ is Artinian, then $\Gamma_{\text {Ann }}(R)$ is a complete graph.

To prove Theorem 10, we need two next lemmas.

Lemma 7. Assume that $R$ is a non-reduced Noetherian ring with $\operatorname{depth}(R)=$ 0 . Then one of the following statements holds:

(i) $\operatorname{Ann}(\mathfrak{m}) \subseteq \mathfrak{m}$ for every $\mathfrak{m} \in \operatorname{Max}(R)$.

(ii) $R \cong F_{1} \oplus \cdots \oplus F_{k} \oplus S$, where every $F_{i}$ is a field, for $1 \leq i \leq k$, and $S$ is a ring such that $\operatorname{Ann}(\mathfrak{m}) \subseteq \mathfrak{m}$ for every $\mathfrak{m} \in \operatorname{Max}(S)$.

Proof. Since $\operatorname{depth}(R)=0,[4$, Proposition 1.2.1] implies that $\operatorname{Ann}(\mathfrak{m}) \neq\{0\}$, for every $\mathfrak{m} \in \operatorname{Max}(R)$. By [11, Corollary 9.36] and the prime avoidance theorem (see [11, Theorem 3.61 ]), $|\operatorname{Max}(R)|<\infty$. Let $|\operatorname{Max}(R)|=n$ for some positive integer $n$. If $\operatorname{Ann}(\mathfrak{m}) \subseteq \mathfrak{m}$ for all maximal ideals $\mathfrak{m}$ in $R$, then the proof is complete. Now, suppose that there exists a maximal ideal $\mathfrak{m}$ in $R$ such that $\operatorname{Ann}(\mathfrak{m}) \nsubseteq \mathfrak{m}$. So, $\operatorname{Ann}(\mathfrak{m})+\mathfrak{m}=R$ and hence, by [11, Lemma 3.58], $\operatorname{Ann}(\mathfrak{m}) \cap \mathfrak{m}=\operatorname{Ann}(\mathfrak{m}) \mathfrak{m}=0$. By Chinese remainder theorem (see [11, Exercise $3.60]), R \cong R / \mathfrak{m} \oplus R / \operatorname{Ann}(\mathfrak{m})$. It is not hard to see that $R / \operatorname{Ann}(\mathfrak{m})$ is also Noetherian, $\operatorname{depth}(R / \operatorname{Ann}(\mathfrak{m}))=0$ and $R / \operatorname{Ann}(\mathfrak{m})$ has $n-1$ maximal ideals. Finally, by induction on $n$, the result holds.

Lemma 8. Let $R$ be a Noetherian ring. Then the subgraph induced by nilpotent ideals of $R$ is complete.

Proof. Suppose that $I$ and $J$ are two distinct non-zero nilpotent ideals of $R$. Thus there exists $n \in \mathbb{N}$ such that $J^{n}=\{0\}$ and $J^{n-1} \neq\{0\}$. If $I J=\{0\}$, then $I$ is adjacent to $J$. Hence we may assume that $I J \neq\{0\}$. If $I J^{n-1} \neq\{0\}$, then $I \cap \operatorname{Ann}(J) \neq\{0\}$ and hence $I$ is adjacent to $J$. Otherwise, if $I J^{n-1}=\{0\}$, then $J \cap \operatorname{Ann}(I) \neq\{0\}$ and hence $I$ is adjacent to $J$. This completes the proof.

The following remark will be used frequently in this paper. 
Remark 9. It is well known that a commutative $\operatorname{ring} R$ is Noetherian and $\operatorname{soc}(R) \leq_{e} R$ if and only if $R$ is Artinian. (This is not true for arbitrary right Noetherian rings.) The interested reader may find a generalization of this fact for every unitary (not necessarily commutative) ring in [5, Theorem 2.5].

Theorem 10. Let $R$ be a ring.

(i) If $R$ is an Artinian ring, then $\Gamma_{\mathrm{Ann}}(R)$ is a complete graph.

(ii) Conversely, if $R$ is non-reduced, Noetherian, $\operatorname{soc}(\operatorname{Nil}(R)) \leq_{e} \operatorname{Nil}(R)$ and $\Gamma_{\text {Ann }}(R)$ is a complete graph, then $R$ is an Artinian ring.

Proof. (i) Suppose that $R$ is an Artinian ring. Then by [11, Exercise 8.50], $R \cong R_{1} \times \cdots \times R_{n}$, where $R_{i}$ is an Artinian local ring with maximal ideal $\mathfrak{m}_{i}$ for every $1 \leq i \leq n$. If $n=1$, then Lemma 8 implies that $\Gamma_{\text {Ann }}(R)$ is complete. Assume that $n \geq 2$. Let $I=I_{1} \times \cdots \times I_{n}$ and $J=J_{1} \times \cdots \times J_{n}$ be two non-trivial distinct ideals of $R$. Since $I$ and $J$ are distinct, there exists $k \in\{1, \ldots, n\}$ such that $I_{k} \neq J_{k}$. If $J_{k}=R_{k}$, then $J \cap \operatorname{Ann}(I) \neq\{0\}$ and so $I$ is adjacent to $J$. If $J_{k}=\{0\}$, then $I \cap \operatorname{Ann}(J) \neq\{0\}$ and so $I$ and $J$ are adjacent. If $I_{k}$ and $J_{k}$ are non-trivial ideals of $R_{k}$, then since $I_{k}$ is adjacent to $J_{k}$ in $\Gamma_{\text {Ann }}\left(R_{k}\right)$, we have $J \cap \operatorname{Ann}(I) \neq\{0\}$ or $I \cap \operatorname{Ann}(J) \neq\{0\}$ and hence $I$ and $J$ are adjacent in $\Gamma_{\text {Ann }}(R)$, as desired.

(ii) Suppose that $\Gamma_{\mathrm{Ann}}(R)$ is a complete graph. If $R$ contains a regular element, say $x$, then it is easy to see that $R x$ is not adjacent to $R x^{2}$ in $\Gamma_{\text {Ann }}(R)$, a contradiction. Thus $\operatorname{depth}(R)=0$, and hence [4, Proposition 1.2.1] implies that $\operatorname{Ann}(\mathfrak{m}) \neq\{0\}$, for every $\mathfrak{m} \in \operatorname{Max}(R)$. By the prime avoidance theorem (see [11, Theorem 3.61 ]) and [11, Corollary 9.36], $R$ contains a finite number of maximal ideals. Thus by Lemma 7 , with no loss of generality, one can suppose that $\operatorname{Ann}(\mathfrak{m}) \subseteq \mathfrak{m}$ for all $\mathfrak{m} \in \operatorname{Max}(R)$. Let $\operatorname{Max}(R)=\left\{\mathfrak{m}_{1}, \ldots, \mathfrak{m}_{n}\right\}$. We claim that $\mathfrak{m}_{i} \leq_{e} R$ for every $1 \leq i \leq n$. Suppose to the contrary, there exists $1 \leq i \leq n$ such that $\mathfrak{m}_{i}$ is not essential in $R$. Hence $\mathfrak{m}_{i} \cap I=\{0\}$ for some non-zero ideal $I$ of $R$. As $\operatorname{Ann}\left(\mathfrak{m}_{i}\right) \subseteq \mathfrak{m}_{i}, \operatorname{Ann}\left(\mathfrak{m}_{i}\right) \cap I=\{0\}$. This implies that $I \subseteq \operatorname{Ann}\left(\operatorname{Ann}\left(\mathfrak{m}_{i}\right)\right)=\mathfrak{m}_{i}$ which is a contradiction and so the claim is proved. By $[13,17.3], \mathrm{J}(R)=\bigcap_{i=1}^{n} \mathfrak{m}_{i} \leq_{e} R$. Hence $\operatorname{soc}(R) \subseteq \mathrm{J}(R)$. If $K$ is a minimal ideal of $R$, then either $K^{2}=0$ or $K=e R$ for some idempotent $e \in K$. Now $K=e R$ implies that $K=0$, by [11, Lemma 3.17], because $\operatorname{soc}(R) \subseteq \mathrm{J}(R)$. Hence $K^{2}=0$ and $K \subseteq \operatorname{Nil}(R)$. Thus $\operatorname{soc}(R) \subseteq \operatorname{Nil}(R)$. On the other hand, [13, 21.2] implies that $\operatorname{soc}(\operatorname{Nil}(R))=\operatorname{soc}(R) \cap \operatorname{Nil}(R)$. Thus $\operatorname{soc}(\operatorname{Nil}(R))=\operatorname{soc}(R)$ and $\operatorname{soc}(R) \leq_{e} \operatorname{Nil}(R)$. To complete the proof, we prove that $\operatorname{Nil}(R) \leq_{e} R$ (see Remark 9 and $[13,17.3])$. Since $\operatorname{Nil}(R)=\bigcap_{\mathfrak{p} \in \operatorname{Min}(R)} \mathfrak{p}$ and $|\operatorname{Min}(R)|<\infty$, it suffices to show that $\mathfrak{p} \leq_{e} R$ for every $\mathfrak{p} \in \operatorname{Min}(R)$. To see this, suppose $I \cap \mathfrak{p}=\{0\}$ for some non-zero ideal $I$ of $R$. We next show that $I \cap \operatorname{Ann}(I)=\{0\}$. Let $0 \neq x \in I \cap \operatorname{Ann}(I)$. Then $x y=0$ for every $y$ in $I$. This implies that $x \in \mathfrak{p}$ or $y \in \mathfrak{p}$, a contradiction. Hence $I \cap \operatorname{Ann}(I)=\{0\}$. Also, $\operatorname{soc}(R) \subseteq \operatorname{Nil}(R) \subseteq \mathfrak{p}$ and $I \cap \mathfrak{p}=\{0\}$ imply that $I$ is not a minimal ideal. Therefore, there exists $J \in \mathbb{I}(R)$ such that $J \varsubsetneqq I$. Thus $J \cap \operatorname{Ann}(I)=\{0\}$. Also, since $\operatorname{Ann}(J) \subseteq \mathfrak{p}$, 
we conclude that $I \cap \operatorname{Ann}(J)=\{0\}$. Now, $J \cap \operatorname{Ann}(I)=I \cap \operatorname{Ann}(J)=\{0\}$ shows that $I$ and $J$ are not adjacent, a contradiction. Thus $\mathfrak{p} \leq_{e} R$.

Next, we study reduced rings whose annihilator ideal graphs are complete.

Theorem 11. Let $R$ be a reduced ring. Then $\Gamma_{\mathrm{Ann}}(R)$ is a complete graph if and only if $\operatorname{Ann}(I) \neq \operatorname{Ann}(J)$ for every distinct pair $I, J \in \mathbb{I}(R)$.

Proof. Suppose that $\Gamma_{\mathrm{Ann}}(R)$ is a complete graph and $\operatorname{Ann}(I)=\operatorname{Ann}(J)$, for some $I, J \in \mathbb{I}(R)$. Then we have $I \cap \operatorname{Ann}(J) \neq\{0\}$ or $J \cap \operatorname{Ann}(I) \neq\{0\}$ and hence $I \cap \operatorname{Ann}(I) \neq\{0\}$ or $J \cap \operatorname{Ann}(J) \neq\{0\}$, which is a contradiction, as $R$ is reduced. To prove the converse, assume that $I$ is an arbitrary vertex of $\Gamma_{\text {Ann }}(R)$ and $\operatorname{Ann}(I) \neq \operatorname{Ann}(K)$, for every $K \in \mathbb{I}(R) \backslash\{I\}$. We show that $I$ is adjacent to every other vertex. Assume to the contrary, $I$ is not adjacent to $J$, for some $J \in \mathbb{I}(R)$. Thus $I \cap \operatorname{Ann}(J)=\{0\}$ and $J \cap \operatorname{Ann}(I)=\{0\}$ and hence $I \operatorname{Ann}(J)=J \operatorname{Ann}(I)=\{0\}$. Therefore, $\operatorname{Ann}(I)=\operatorname{Ann}(J)$, a contradiction. Since $I$ is arbitrary, we deduce that $\Gamma_{\mathrm{Ann}}(R)$ is complete.

Theorem 12. Let $R$ be a Noetherian reduced ring. Then $\Gamma_{\text {Ann }}(R)$ is a complete graph if and only if $R$ is a direct product of finitely many fields.

Proof. One side is clear. To prove the other side, suppose that $\Gamma_{\mathrm{Ann}}(R)$ is a complete graph. If $R$ contains a regular element, say $x$, then it is easy to see that $R x$ is not adjacent to $R x^{2}$ in $\Gamma_{\mathrm{Ann}}(R)$, a contradiction. Thus $\operatorname{depth}(R)=0$ and so it follows from [4, Proposition 1.2.1] that $\operatorname{Ann}(I) \neq\{0\}$ for every $I \in \mathbb{I}(R)$. Let $\mathfrak{m} \in \operatorname{Max}(R)$. Then $\mathfrak{m} \subseteq Z(R)=\bigcup_{\mathfrak{p} \in \operatorname{Min}(R)} \mathfrak{p}$, by [8, Corollary 2.4]. It follows from the prime avoidance theorem (see [11, Theorem 3.61]) that there exists $\mathfrak{p} \in \operatorname{Min}(R)$ such that $\mathfrak{m}=\mathfrak{p}$ and so $R$ is Artinian, by [11, Corollary 8.45]. Since $R$ is reduced, [11, Exercise 8.50] completes the proof.

In the next proposition, we study the subgraph induced by prime ideals of a ring $R$.

Proposition 13. Let $R$ be a ring. Then the subgraph induced by prime ideals of $R$ is the join of a complete graph and a totally disconnected graph.

Proof. Put $\Lambda:=\{\mathfrak{p} \in \operatorname{Spec}(R): \operatorname{Ann}(\mathfrak{p}) \neq\{0\}\}$ and $\Sigma:=\{\mathfrak{p} \in \operatorname{Spec}(R)$ : $\operatorname{Ann}(\mathfrak{p})=\{0\}\}$. Clearly, the subgraph induced by the vertex-set $\Sigma$ is the totally disconnected graph $\bar{K}_{|\Sigma|}$. We show that the subgraph induced by the vertex-set $\Lambda$ is complete. Assume that $\mathfrak{p}_{1}, \mathfrak{p}_{2} \in \Lambda$ are distinct. $\operatorname{As} \mathfrak{p}_{1} \operatorname{Ann}\left(\mathfrak{p}_{1}\right)=\{0\}$, we have either $\mathfrak{p}_{1} \subseteq \mathfrak{p}_{2}$ or $\operatorname{Ann}\left(\mathfrak{p}_{1}\right) \subseteq \mathfrak{p}_{2}$. If $\operatorname{Ann}\left(\mathfrak{p}_{1}\right) \subseteq \mathfrak{p}_{2}$, then $\mathfrak{p}_{1}$ is adjacent to $\mathfrak{p}_{2}$. Otherwise, we may assume that $\mathfrak{p}_{1} \subseteq \mathfrak{p}_{2}$. Since $\mathfrak{p}_{2} \operatorname{Ann}\left(\mathfrak{p}_{2}\right)=\{0\}$, one can easily see that $\operatorname{Ann}\left(\mathfrak{p}_{2}\right) \subseteq \mathfrak{p}_{1}$ and so $\mathfrak{p}_{1}$ is adjacent to $\mathfrak{p}_{2}$. Thus the subgraph induced by the vertex-set $\Lambda$ is complete. To complete the proof, we show that every vertex of $\Lambda$ is adjacent to all vertices of $\Sigma$. Let $\mathfrak{p}_{1} \in \Lambda$ and $\mathfrak{p}_{2} \in \Sigma$. If $\operatorname{Ann}\left(\mathfrak{p}_{1}\right) \cap \mathfrak{p}_{2}=\{0\}$, then $\operatorname{Ann}\left(\mathfrak{p}_{1}\right) \mathfrak{p}_{2}=\{0\}$ and this contradicts the assumption $\operatorname{Ann}\left(\mathfrak{p}_{2}\right)=\{0\}$. Hence $\operatorname{Ann}\left(\mathfrak{p}_{1}\right) \cap \mathfrak{p}_{2} \neq\{0\}$ and so $\mathfrak{p}_{1}$ is adjacent to $\mathfrak{p}_{2}$. So, $\Gamma_{\mathrm{Ann}}(R)[\operatorname{Spec}(R)]=\bar{K}_{|\Sigma|} \vee K_{|\Lambda|}$. 


\section{Clique number and coloring of an annihilator ideal graph}

In this section, we investigate the clique and chromatic numbers of $\Gamma_{\mathrm{Ann}}(R)$. By Part (i) of Theorem 10, if $R$ is an Artinian ring, then $\Gamma_{\text {Ann }}(R)$ is complete. So, we study the clique and chromatic numbers of $\Gamma_{\text {Ann }}(R)$, when $R$ is nonArtinian. First, we determine the clique number and the chromatic number of $\Gamma_{\text {Ann }}(R)$, where $R$ is a non-Artinian ring and is a direct sum of finitely many integral domains. Also, it is proved that if $R$ is a Noetherian ring and $\omega\left(\Gamma_{\text {Ann }}(R)\right)=2$, then $R$ is a Gorenstein ring.

Theorem 14. Let $R$ be a non-Artinian ring such that $R \cong D_{1} \oplus \cdots \oplus D_{n}$, where $D_{i}$ is an integral domain, for every $1 \leq i \leq n$. Then $\omega\left(\Gamma_{\text {Ann }}(R)\right)=$ $\chi\left(\Gamma_{\text {Ann }}(R)\right)=2^{n}-1$.

Proof. Let $I, J \in \mathbb{I}(R)$ and $\sim$ be an equivalence relation on $\mathbb{I}(R)$. So there exist ideals $I_{i}$ and $J_{i}$ of $D_{i}$, such that $I=I_{1} \times \cdots \times I_{n}$ and $J=J_{1} \times \cdots \times J_{n}$, for all $1 \leq i \leq n$. We define $I \sim J$ if, " $I_{i}=\{0\}$ if and only if $J_{i}=\{0\}$ ", for all $1 \leq i \leq n$. We denote by $[X]=\{Y \in \mathbb{I}(R) \mid Y \sim X\}$ the equivalence class of $X$. Let $X=I_{1} \times \cdots \times I_{n}$ be an arbitrary ideal of $\mathbb{I}(R)$. Then for every $I_{i}$, we have $I_{i}=\{0\}$ or $I_{i} \neq\{0\}$, for every $1 \leq i \leq n$. Hence every $I_{i}$ has two choices. Therefore, the number of all this selections for $X$ is $2^{n}-1$. This implies that the number of equivalence classes is $2^{n}-1$. Now, suppose that $[X]$ and $[Y]$ are two distinct arbitrary equivalence classes. We show that there is no adjacency between two elements of $[X]$ (in $\Gamma_{\text {Ann }}(R)$ ) and each element of $[X]$ is adjacent to each element of $[Y]$. To see this, let $I$ and $J$ be two elements of $[X]$ and $K$ be an element of $[Y]$. So there exist ideals $I_{i}, J_{i}$ and $K_{i}$ of $D_{i}$, such that $I=I_{1} \times \cdots \times I_{n}, J=J_{1} \times \cdots \times J_{n}$ and $K=K_{1} \times \cdots \times K_{n}$. Thus $I_{i}=\{0\}$ if and only if $J_{i}=\{0\}$, for all $1 \leq i \leq n$. Since $D_{i}$ is an integral domain, $I$ is not adjacent to $J$. On the other hand, $I \nsim K$, so $I_{i}=\{0\}$ and $K_{i} \neq\{0\}$ for some $1 \leq i \leq n$. Hence for each $0 \neq a \in K_{i}$ we have $0 \times \cdots \times 0 \neq 0 \times \cdots \times 0 \times a \times 0 \times \cdots \times 0 \in K$. Clearly, $0 \times \cdots \times 0 \times a \times 0 \times \cdots \times 0 I=\{0\}$. So $I$ is adjacent to $K$. Indeed, $\Gamma_{\text {Ann }}(R)$ is a complete $\left(2^{n}-1\right)$-partite graph. Thus $\omega\left(\Gamma_{\text {Ann }}(R)\right)=\chi\left(\Gamma_{\text {Ann }}(R)\right)=2^{n}-1$.

Corollary 15. Let $R$ be a ring such that $R \subseteq D_{1} \oplus \cdots \oplus D_{n}$ and $R \cap D_{i} \neq\{0\}$, for all $1 \leq i \leq n$, where $D_{i}$ is an integral domain. Then $\omega\left(\Gamma_{\mathrm{Ann}}(R)\right)=$ $\chi\left(\Gamma_{\text {Ann }}(R)\right)=2^{n}-1$.

Example 16. Let $R \cong D_{1} \oplus D_{2}$, where $D_{1}$ and $D_{2}$ are integral domains. We put $A:=\left\{I \mid I\right.$ is an ideal of $R$ and $\left.I=I_{1} \times(0), I_{1} \neq(0)\right\}, B:=\{I \mid I$ is an ideal of $R$ and $\left.I=(0) \times I_{2}, I_{2} \neq(0)\right\}$ and $C:=\{I \mid I$ is an ideal of $R$ and $\left.I=I_{1} \times I_{2}, I \neq R, I_{1} \neq(0), I_{2} \neq(0)\right\}$. Clearly, $\mathbb{I}(R)=A \cup B \cup C$, and $A \cap B=A \cap C=B \cap C=\varnothing$. Now, let $I, J \in A(\in B$ or $\in C)$ and $K \in B$ or $K \in C(\in A)$. Then, it is easy to check that there is no adjacency between two vertices $I$ and $J$, but $I$ is adjacent to $K$. This implies that $\Gamma_{\text {Ann }}(R)$ is a complete 3 -partite graph and $\omega\left(\Gamma_{\text {Ann }}(R)\right)=\chi\left(\Gamma_{\text {Ann }}(R)\right)=3$ (see Figure 1$)$. 


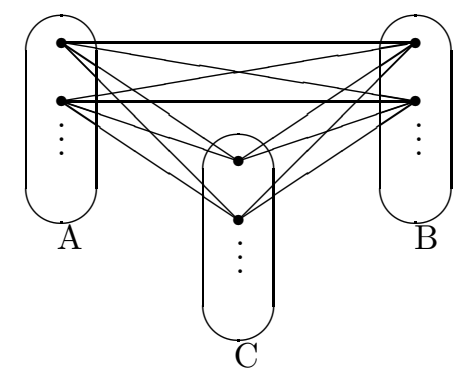

Figure 1. A part of $\Gamma_{\text {Ann }}\left(D_{1} \oplus D_{2}\right)$.

Let $R$ be a Noetherian local ring. Then $R$ is said to be Cohen-Macaulay ring if $\operatorname{depht}(R)=\operatorname{dim}(R)$. In general, if $R$ is a Noetherian ring, then $R$ is a Cohen-Macaulay ring if $R_{\mathfrak{m}}$ is a Cohen-Macaulay ring, for all maximal ideals $\mathfrak{m}$, where $R_{\mathfrak{m}}$ is the localization of $R$ at $\mathfrak{m}$. Also, a Noetherian local ring $R$ is called Gorenstein if $R$ is Cohen-Macaulay and $\operatorname{dim}_{R / \mathfrak{m}}(\operatorname{soc}(R))=1$, where $\mathfrak{m}$ is the unique maximal ideal of $R$. In general, if $R$ is a Noetherian ring, then $R$ is a Gorenstein ring if $R_{\mathfrak{m}}$ is a Gorenstein ring, for all maximal ideals $\mathfrak{m}$. In the next theorem, we study the special case when $R$ is a Gorenstein ring (see [4]).

Theorem 17. Let $R$ be a Noetherian ring. If $\omega\left(\Gamma_{\mathrm{Ann}}(R)\right)=2$, then $R$ is a Gorenstein ring.

Proof. If $R$ is an Artinian ring, then by Part (i) of Theorem 10, $\Gamma_{\text {Ann }}(R)$ is a complete graph. Since $\omega\left(\Gamma_{\mathrm{Ann}}(R)\right)=2$, we have $|\mathbb{I}(R)|=2$. Now, if $R$ is a local ring, then clearly $R$ is a Gorenstein ring (see [4, Exercise 3.2.15]). Also, if $R$ is a non-local ring, then by [11, Exercise 8.50], $R \cong R_{1} \times R_{2}$, where $R_{i}$ is a field for $i=1,2$. This implies that $R$ is a Gorenstein ring. Hence, we may suppose that $R$ is not Artinian and we try to find a contradiction. If $R$ is a reduced ring, then by [8, Corollary 2.4], we have $Z(R)=$ $\bigcup_{\mathfrak{p} \in \operatorname{Min}(R)} \mathfrak{p}$. Let $\operatorname{Min}(R)=\left\{\mathfrak{p}_{1}, \ldots, \mathfrak{p}_{k}\right\}$. In this case if $|\operatorname{Min}(R)| \geq 3$, then $\mathfrak{p}_{2} \mathfrak{p}_{3} \cdots \mathfrak{p}_{k}-\mathfrak{p}_{1} \mathfrak{p}_{3} \cdots \mathfrak{p}_{k}-\mathfrak{p}_{2} \mathfrak{p}_{3} \cdots \mathfrak{p}_{k-1}-\mathfrak{p}_{2} \mathfrak{p}_{3} \cdots \mathfrak{p}_{k}$ is a triangle in $\Gamma_{\text {Ann }}(R)$ (see [11, Lemma 3.55]), which is a contradiction. Now if $\operatorname{Min}(R)=\left\{\mathfrak{p}_{1}, \mathfrak{p}_{2}\right\}$, then by [11, Theorem 3.61], one can easily see that either $R$ is an Artinian ring or $\mathfrak{p}_{1}-R x-\mathfrak{p}_{2}-\mathfrak{p}_{1}$ is a triangle in $\Gamma_{\mathrm{Ann}}(R)$, for some regular element $x$ in $R$, a contradiction. Hence we may assume that $R$ is not reduced. We claim that if $\operatorname{depth}(R)=0$, then $\omega\left(\Gamma_{\mathrm{Ann}}(R)\right)=\infty$. Since $R$ is Noetherian and $\operatorname{depth}(R)=0$, it follows from [4, Proposition 1.2.1] that $\operatorname{Ann}(I) \neq\{0\}$, for every $I \in \mathbb{I}(R)$. If the number of essential ideals of $R$ is finite, then $[13,21.1]$ implies that $\operatorname{soc}(R) \leq_{e} R$ and hence $R$ is Artinian, by Remark 9 , which is impossible. Thus $R$ has infinitely many essential ideals. Since $\operatorname{Ann}(I) \neq\{0\}$, for every $I \in \mathbb{I}(R)$, we conclude that $\omega\left(\Gamma_{\mathrm{Ann}}(R)\right)=\infty$ and so the claim is proved. Thus 
$\operatorname{depth}(R) \neq 0$. If for all ideals $I \subseteq Z(R), I=\operatorname{Ann}(I)$, then $Z(R)=\operatorname{Nil}(R)$. Now we show that $Z(R)$ is not a minimal ideal of $R$. Suppose to the contrary, $Z(R)$ is a minimal ideal of $R$. Then $Z(R)$ is the unique minimal ideal of $R$ and $\operatorname{soc}(R)=Z(R)$. Thus by Remark $9, R$ is an Artinian ring, which is not true. Since $Z(R)$ is not a minimal ideal of $R, Z(R)-I-R x-Z(R)$ is a triangle in $\Gamma_{\text {Ann }}(R)$, where $x$ is a regular element in $R$ and $I \subseteq Z(R)$ is a non-zero ideal of $R$, a contradiction. Now suppose that there exists $I \in \mathbb{I}(R)$ such that $I \subseteq Z(R)$ and $I \neq \operatorname{Ann}(I)$. If $x$ is a regular element of $R$, then $I-\operatorname{Ann}(I)-R x-I$ is a triangle in $\Gamma_{\text {Ann }}(R)$, which is impossible. This completes the proof.

The following corollary is an immediate consequence of Theorem 17.

Corollary 18. Let $R$ be a Noetherian ring and $\Gamma_{\mathrm{Ann}}(R)$ is a forest. Then we have the following statements:

(i) $R$ is an integral domain.

(ii) $R$ is a Gorenstein ring.

In the next theorem, we study the special case that $\Gamma_{\mathrm{Ann}}(R)$ is a star graph.

Theorem 19. Let $R$ be a ring such that $\operatorname{depth}(R) \neq 0$. Then the following statements are equivalent:

(i) $Z(R) \neq\{0\}$ is a minimal ideal of $R$.

(ii) $\Gamma_{\mathrm{Ann}}(R)$ is a star graph.

Proof. First suppose that $Z(R) \neq\{0\}$ is a minimal ideal of $R$. Thus we have $\operatorname{Ann}(Z(R)) \neq\{0\}$. Let $I, J \in \mathbb{I}(R) \backslash\{Z(R)\}$. Since $\operatorname{depth}(R) \neq 0, I$ and $J$ contain regular elements. Hence $I$ is not adjacent to $J$, and they are adjacent to $Z(R)$. Therefore, $\Gamma_{\mathrm{Ann}}(R)$ is a star graph.

Conversely, suppose that $\Gamma_{\text {Ann }}(R)$ is a star graph. Thus by Lemma 1, $Z(R) \neq\{0\}$. Now, let $x$ be a regular element of $R$ and $y \neq 0$ be an arbitrary element in $Z(R)$. Therefore, there exists $0 \neq z \in Z(R)$ such that $z y=0$. So, it is easy to see that $R x-R y-R z-R x$ is a triangle in $\Gamma_{\text {Ann }}(R)(R x$ is an essential ideal), which is a contradiction. Also, $R x \neq R y$ and $R x \neq R z$. Thus $R z=R y$ and so there exists an element $r \in R$ such that $y=r z$. Hence $y^{2}=r z y=0$. This implies that $Z(R)=\operatorname{Nil}(R)$. Next we show that $\operatorname{Nil}(R)$ is a minimal ideal of $R$. To see this, we show that $\operatorname{Nil}(R)=R y$ for every $y \in \operatorname{Nil}(R)$. Let $\operatorname{Nil}(R)=R y+R z+\cdots$. If $y z=0$, since $R x$ is essential, we deduce that $R y=R z$ and if $y z \neq 0$, then $y z \in R y$ and $y z R z=\{0\}$, so similarly $R y=R z$. Therefore, $\operatorname{Nil}(R)=R y$ for every $y \in \operatorname{Nil}(R)$, as desired.

In the above theorem, $R$ can not be a Noetherian ring. Because if $R$ is Noetherian, then $R$ has exactly one minimal ideal. Thus $\operatorname{soc}(R) \leq_{e} R$ and hence by Remark 9, $R$ is an Artinian ring. Thus $\operatorname{depth}(R)=0$, which is impossible.

In the last result of this section, we show that the number of annihilator ideals of $R$ is at most $2^{|\operatorname{Min}(R)|}$. 
Proposition 20. Let $R$ be a reduced ring and $\omega\left(\Gamma_{\mathrm{Ann}}(R)\right)<\infty$. Then the number of annihilator ideals of $R$ is finite.

Proof. Let $\left\{x_{i}\right\}_{i \in \mathbb{N}}$ be an infinite clique in $\Gamma(R)$. Then $\left\{R x_{i}\right\}_{i \in \mathbb{N}}$ is an infinite clique in $\Gamma_{\mathrm{Ann}}(R)$, which is impossible. Hence $\omega(\Gamma(R))<\infty$. Now by $[3$, Theorems 3.7 and 3.8], we may assume that $\operatorname{Min}(R)=\left\{\mathfrak{p}_{1}, \ldots, \mathfrak{p}_{k}\right\}$. Suppose that $I \in \mathbb{I}(R)$. Then since $\operatorname{Ann}(I) \operatorname{Ann}(\operatorname{Ann}(I))=\{0\}$, we have either $\operatorname{Ann}(I) \subseteq$ $\mathfrak{p}_{i}$ or $\operatorname{Ann}(\operatorname{Ann}(I)) \subseteq \mathfrak{p}_{i}$, for every $1 \leq i \leq k$. Now, we consider two following sets:

$$
\Delta:=\left\{i: 1 \leq i \leq k, \operatorname{Ann}(I) \subseteq \mathfrak{p}_{i}\right\}, \quad \Omega:=\left\{i: 1 \leq i \leq k, \operatorname{Ann}(\operatorname{Ann}(I)) \subseteq \mathfrak{p}_{i}\right\} .
$$

Clearly $\Delta \cup \Omega=\{1,2, \ldots, k\}$. Now, since $R$ is reduced and $\operatorname{Ann}(\operatorname{Ann}(\operatorname{Ann}(I)))$ $=\operatorname{Ann}(I)$, we have $\operatorname{Ann}(I) \subseteq \bigcap_{i \in \Delta} \mathfrak{p}_{i} \subseteq \operatorname{Ann}\left(\bigcap_{i \in \Omega} \mathfrak{p}_{i}\right) \subseteq \operatorname{Ann}(I)$. Thus the number of annihilator ideals of $R$ is finite.

\section{Some finiteness conditions in annihilator ideal graphs}

In this section, we study some conditions under which $\Gamma_{\mathrm{Ann}}(R)$ is a finite graph. For instance, we show that if a minimal ideal of $R$ has a finite degree in $\Gamma_{\text {Ann }}(R)$, then $\Gamma_{\text {Ann }}(R)$ is finite. Also, it is proved that if $R$ is a reduced ring and $|\operatorname{Min}(R)|<\infty$, then $\alpha\left(\Gamma_{\mathrm{Ann}}(R)\right)<\infty$ if and only if $R$ is a direct product of finitely many fields.

Proposition 21. If a minimal ideal of $R$ has a finite degree in $\Gamma_{\mathrm{Ann}}(R)$, then $\Gamma_{\mathrm{Ann}}(R)$ is a finite graph.

Proof. Suppose that $I$ is a minimal ideal of $R$ such that $\operatorname{deg}(I)<\infty$. Also, let $J$ be an ideal of $R$. Then either $I \subseteq J$ or $I \cap J=\{0\}$. Put

$$
\Lambda:=\{J: J \text { is a non-trivial ideal of } R \text { and } I \cap J=\{0\}\}
$$

and

$$
\Sigma:=\{J: J \text { is a non-trivial ideal of } R \text { and } I \varsubsetneqq J\} .
$$

We show that $\Lambda \cup \Sigma$ is finite. Clearly, $I$ is adjacent to all vertices of $\Lambda$ and since $\operatorname{deg}(I)<\infty, \Lambda$ is finite. Now if $I^{2}=\{0\}$, then $I$ is adjacent to all vertices of $\Sigma$, and since $\operatorname{deg}(I)<\infty, \Sigma$ is finite. Otherwise, if $I^{2}=I$, then by Brauer's Lemma (see [9, 10.22]), $R=R e \oplus R(1-e$ ), where $e$ is a idempotent element of $R$. Thus we may assume that $R \cong F \times S$, where $F$ is a field and $S$ is a ring. If $I=F \times\{0\}$, then $I$ is adjacent to all vertices of the form $F \times Y$, where $Y$ is a non-trivial ideal in $S$. Since $\operatorname{deg}(I)<\infty, \Sigma$ is finite. Finally, suppose that the minimal ideal $I$ is of the form $I=0 \times J$, where $J$ is a minimal ideal of $S$. Let $L=F \times K$ be a vertex of $\Gamma_{\text {Ann }}(R)$, where $K$ is an ideal of $S$. Obviously, $\operatorname{Ann}(I) \cap L \neq\{0\}$. Since $\operatorname{deg}(I)<\infty$, we deduce that $S$ has finitely many ideals. Therefore, the number of ideals of $R$ is finite, as desired.

Theorem 22. $\Delta\left(\Gamma_{\mathrm{Ann}}(R)\right)<\infty$ if and only if either $R$ is an integral domain or $\Gamma_{\mathrm{Ann}}(R)$ is finite. 
Proof. Assume that $R$ is not an integral domain and $\operatorname{deg}(v)<\infty$ for all $v \in$ $V\left(\Gamma_{\text {Ann }}(R)\right)$. Let $x \in Z(R) \backslash\{0\}$ and set $I:=R x$ and $J:=\operatorname{Ann}(x)$. If $I$ is not Artinian as an $R$-module, then there exists a non-stationary chain of submodules of $I$, say $I \supsetneqq I_{1} \supsetneqq I_{2} \supsetneqq \cdots$. Thus $\operatorname{deg}(J)=\infty$, which is a contradiction. Hence $I$ is an Artinian $R$-module. Similarly, $J$ is an Artinian $R$-module. Since $I \cong_{R} R / J$ ( $R$-module isomorphism), we conclude that $R / J$ is an Artinian $R$-module. By [11, Corollary 7.19], $R$ is an Artinian ring. Hence Part (i) of Theorem 10 implies that the number of ideals of $R$ is finite. The converse is clear.

Theorem 23. Let $R$ be a Cohen-Macaulay ring. Then $\alpha\left(\Gamma_{\mathrm{Ann}}(R)\right)<\infty$ if and only if $R$ is an Artinian ring.

Proof. Suppose that $\alpha\left(\Gamma_{\text {Ann }}(R)\right)<\infty$. We show that $\operatorname{depth}(R)=0$. Assume to the contrary, $\operatorname{depth}(R) \neq 0$. If $x$ is a regular element in $R$, then the subgraph induced by the vertex set $\left\{R x^{i}\right\}_{i \in \mathbb{N}}$ is totally disconnected and hence $\alpha\left(\Gamma_{\text {Ann }}(R)\right)=\infty$, which is a contradiction. Thus $\operatorname{depth}(R)=0$ and so $R$ is an Artinian ring. Conversely, assume that $R$ is an Artinian ring. Then by Part (i) of Theorem 10, $\Gamma_{\mathrm{Ann}}(R)$ is complete and hence $\alpha\left(\Gamma_{\mathrm{Ann}}(R)\right)=1$.

We close this paper with the following result.

Theorem 24. Let $R$ be a reduced ring and $|\operatorname{Min}(R)|<\infty$. Then $\alpha\left(\Gamma_{\mathrm{Ann}}(R)\right)<$ $\infty$ if and only if $R$ is a direct product of finitely many fields.

Proof. Suppose that $x$ is a regular element of $R$. Then the subgraph induced by the vertex set $\left\{R x^{i}\right\}_{i \in \mathbb{N}}$ is totally disconnected and hence $\alpha\left(\Gamma_{\text {Ann }}(R)\right)=$ $\infty$, which is a contradiction. Therefore, every non-unit element of $R$ is zerodivisor. If $\mathfrak{m}$ is a maximal ideal of $R$, then $\mathfrak{m} \subseteq Z(R)=\bigcup_{\mathfrak{p} \in \operatorname{Min}(R)} \mathfrak{p}$, by $[8$, Corollary 2.4]. Thus the prime avoidance theorem (see [11, Theorem 3.61]) implies that there exists $\mathfrak{p} \in \operatorname{Min}(R)$ such that $\mathfrak{m}=\mathfrak{p}$. Hence we may assume that $\operatorname{Max}(R)=\left\{\mathfrak{m}_{1}, \ldots, \mathfrak{m}_{n}\right\}$. We show that $\operatorname{soc}(R)=R$. In other word, it is proved that there is no non-trivial essential ideal in $R$ (see $[13,21.1]$ ). Assume to the contrary, $I$ is a non-trivial essential ideal of $R$. Then there exists $\mathfrak{m} \in \operatorname{Max}(R)$ such that $I \subseteq \mathfrak{m}$. Without loss of generality, suppose that $I \subseteq \mathfrak{m}_{1}$. By [11, Lemma 3.55], we have $\bigcap_{i=2}^{n} \mathfrak{m}_{i} \neq\{0\}$. Since $R$ is a reduced ring, $I \cap \mathfrak{m}_{2} \cap \cdots \cap \mathfrak{m}_{n}=\{0\}$, which is a contradiction. Therefore, $\operatorname{soc}(R)=R$ (And so indeed $R$ is semi-simple). This implies that $R$ is a finite direct product of fields (see $[13,20.7]$ ). The converse follows from Part (i) of Theorem 10.

Remark 25. By a similar method to that of Theorem 24, it is easy to see that if $R$ is a Noetherian ring and $\alpha\left(\Gamma_{\text {Ann }}(R)\right)<\infty$, then the number of maximal ideals of $R$ is finite.

Acknowledgements. The authors thank to the referee for his/her careful reading and his/her excellent suggestions. 


\section{References}

[1] S. Akbari, R. Nikandish, and M. J. Nikmehr, Some results on the intersection graphs of ideals of rings, J. Algebra Appl. 12 (2013), no. 4, 1250200, 13 pp.

[2] D. F. Anderson and P. S. Livingston, The zero-divisor graph of a commutative ring, J. Algebra 217 (1999), no. 2, 434-447.

[3] I. Beck, Coloring of commutative rings, J. Algebra 116 (1988), no. 1, 208-226.

[4] W. Bruns and J. Herzog, Cohen-Macaulay Rings, Cambridge University Press, 1997.

[5] J. Chen, N. Ding, and M. F. Yousif, On Noetherian rings with essential socle, J. Aust. Math. Soc. 76 (2004), no. 1, 39-49.

[6] S. Ebrahimi Atani, S. Dolati Pish Hesari, and M. Khoramdel, Total graph of a commutative semiring with respect to identity-summand elements, J. Korean Math. Soc. 51 (2014), no. 3, 593-607.

[7] F. Heydari and M. J. Nikmehr, The unit graph of a left Artinian ring, Acta Math. Hungar. 139 (2013), no. 1-2, 134-146.

[8] J. A. Huckaba, Commutative Rings with Zero-Divisors, Marcel Dekker, Inc., New York, 1988.

[9] T. Y. Lam, A First Course in Non-commutative Rings, Graduate Texts in Mathematics, Vol. 131, Springer-Verlag, Berlin/Heidelberg, New York, 1991.

[10] R. Nikandish and M. J. Nikmehr, The intersection graph of ideals of $\mathbb{Z}_{n}$ is weakly perfect, Util. Math., to appear.

[11] R. Y. Sharp, Steps in Commutative Algebra, Second Edition, London Mathematical Society Student Texts 51, Cambridge University Press, Cambridge, 2000.

[12] D. B. West, Introduction to Graph Theory, Second Edition, Prentice Hall, Upper Saddle River, 2001.

[13] R. Wisbauer, Foundations of Module and Ring Theory, Gordon and Breach Science Publishers, 1991.

Abolfazl Alibemani

FACUlTy OF MATHEMATiCS

K. N. Toosi University of Technology

P.O. BOX 16315-1618, TEHRAN, IrAN

E-mail address: a.alibemani2012@gmail.com

MOHARRAM BAKHTYIARI

Department of Mathematics

College of Basic Sciences

Karaj Branch, Islamic Azad University

AlborZ, Iran

E-mail address: m.bakhtyiari55@gmail.com

REZA NiKANDISH

Department of Mathematics

Jundi-Shapur University of TeChNology

P.O. BOX 64615-334, DEZFul, IrAN

E-mail address: r.nikandish@jsu.ac.ir

MOHAmmad JaVAD Nikmehr

FACULTY OF MATHEMATICS

K. N. Toosi University of Technology

P.O. BOX 16315-1618, TEHRAN, IrAN

E-mail address: nikmehr@kntu.ac.ir 